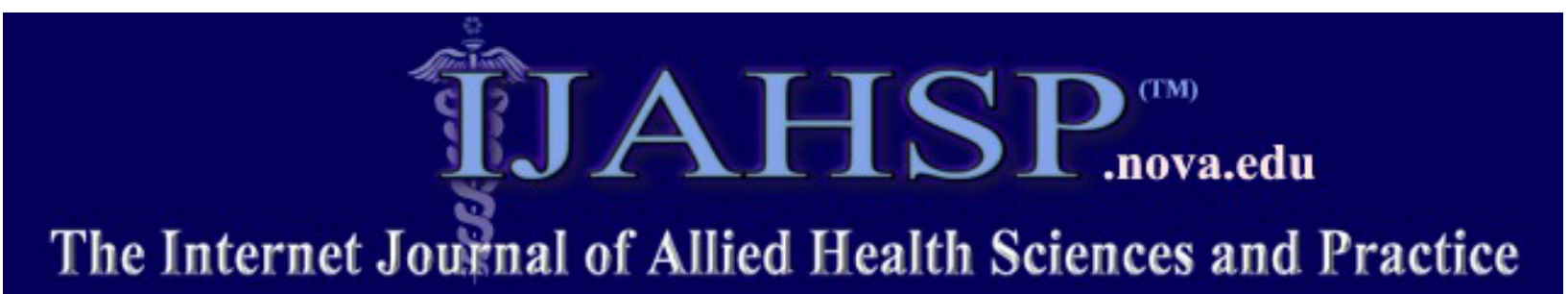

A Peer Reviewed Publication of the College of Health Care Sciences at Nova Southeastern University

Dedicated to allied health professional practice and education

http://ijahsp.nova.edu Vol. 10 No. 4 ISSN 1540-580X

\title{
An Instrument for Assessing Communication Skills of Healthcare and Human Services Students
}

\author{
James Johnston, PhD, R.T. (R) (CV) 1 \\ Laura Fidelie, JD22 \\ Kim W. Robinson, PhD, R.N. ${ }^{3}$ \\ Jeffrey B. Killion, PhD, R.T. (R) $(\mathrm{QM})^{4}$ \\ Phyllis Behrens, $\mathrm{PhD}^{5}$
}

1. Director of Interdisciplinary Education, Associate Professor of Radiologic Sciences, Midwestern State University, Wichita Falls, Texas

2. Assistant Professor of Criminal Justice, Midwestern State University, Wichita Falls, Texas

3. Assistant Professor of Nursing, Midwestern State University, Wichita Falls, Texas

4. Graduate Coordinator, Associate Professor of Radiologic Sciences, Midwestern State University, Wichita Falls, Texas

5. Assistant Professor of Health and Public Administration, Midwestern State University, Wichita Falls, Texas

United States

CITATION: Johnston J, Fidelie L, Robinson K, Killion J, Behrens P. An Instrument for Assessing Communication Skills of Healthcare and Human Services Students. The Internet Journal of Allied Health Sciences and Practice. Oct 2012. Volume 10 Number 4.

\section{ABSTRACT}

This article reports on the original research that developed and tested an instrument to quantify basic communication skills of students in healthcare and human service education programs. Potential uses of this instrument include its use as a pre- and post-test assessment of instruction intended to improve communication skills and/or as another criteria for admission into healthcare and human service education programs. Results of this study indicate that the instrument has value in assessing communication skills.

\section{INTRODUCTION}

Effective communication in healthcare is not just about English as a second language, although that can be a significant issue. ${ }^{1}$ Communication is critical to the quality of care a patient receives from the healthcare team and is vital to the avoidance of medical errors. ${ }^{2}$ Effective communication has been linked to patient satisfaction. ${ }^{3-5}$ Inadequate communication may result in undue mental anguish for both the patient and the patient's family, which is certainly adverse to the overall goals of healthcare. ${ }^{6}$ Similar issues may be present in other human service professions. Research indicates that communication skills do not necessarily improve with experience. ${ }^{7}$ This latter finding in particular indicates a need for identifying and assessing factors that contribute to effective communication in prospective students prior to their admission to a healthcare or human services program. Numerous studies have been identified that evaluate different aspects of communication. This project sought to bring the best of these studies together to formulate an instrument for measuring attributes of effective communication in potential healthcare and human service providers, providing a means of selecting quality students for healthcare and human service programs.

\section{REVIEW OF LITERATURE}




\section{Definitions}

Communication is a multidimensional and complex exchange between people. It may be defined in a variety of ways depending on the setting, context, nature or focus of study, environment, or cultural surroundings. Add to this the ever-changing meaning of some words through slang, or as one author puts it, "plastic words" or words with flexible meaning, and one has a complex issue indeed. ${ }^{8}$ Pei notes that communication is predicated on symbolic values expressed through word, sound, gesture, writing, and drawing. He further states that all accumulated experience transmits between individuals, generations, eras, races, and cultures in some form: spoken, written, gestural, or symbolic. In its broad sense, that being communication, he says language is the hinge of all human activity, as a community of understanding between the producer and receiver of meaning. ${ }^{9}$ The notion of meaning is very important in defining effective communication.

In general, communication encompasses a process of transferring information between sender and receiver through an agreed upon or understood set of symbols, words (language), expressions, or body language. ${ }^{10,11}$ For this study, communication will be defined based upon the end goal of quantifying essential communication skills as one admission criterion for potential healthcare and human services students. Such a definition certainly has overlap with other contexts and settings. Therefore, in this writing, communication is intended to mean the ability to effectively use verbal and nonverbal forms of the English language in a healthcare or human services environment.

\section{Elements of Effective Communication}

To further clarify the concept of effective communication, it is necessary to identify its essential elements. Commonly cited elements of effective verbal communication include active listening techniques such as clarifying and paraphrasing to ensure understanding. ${ }^{12-14} \mathrm{It}$ also includes empathy for the other person's point of view, which is taking a sincere interest in what that person is communicating and attempting to see the situation through his/her eyes.12-14 Establishing a rapport and trust is important for having honest and meaningful communication. ${ }^{12,14,15}$ Another important component is the acknowledgement of prejudices, or how we perceive the other person, and how that may affect our communication with that person. ${ }^{12,13}$

Perhaps the most discussed element of effective communication is awareness of, and an ability to use and interpret, non-verbal communication. ${ }^{12,13,16-18}$ An estimated 60 to 65 percent of a social encounter is communicated nonverbally. Non-verbal communication includes appearance, eye contact, proxemics, facial expressions, and gestures, and varies from one culture to another.13,17,18 Dyche further states that nonverbal communication includes extra textual elements of a face-to-face exchange that can support, modify, or contradict a verbal message. ${ }^{17}$ People of all cultures use nonverbal communication without thinking and it is learned within the environment in which he/she is raised. ${ }^{16}$

Within a healthcare or human services setting, a hierarchical relationship may exist between the participants. For example, a provider in charge of a patient's care may assume a position of authority in that relationship. A similar relationship may exist between a physician and other healthcare providers, a police officer and a citizen, and many others. The added dynamic of authority, although not required in such situations, makes it essential that the person in the position of authority possess the interpersonal skills to create a "safe" and trusting environment for communication to take place.17,18 The level of trust between two parties and their ability to communicate effectively are directly related. ${ }^{12}$ Grover states that when conflicts arise, the ability to negotiate becomes vital for effective communication. ${ }^{12}$ Negotiation skills imply communication skills. She further states that when looking specifically at interdisciplinary communication, it is important to find connections across disciplines and to have respect for the involved disciplines.

\section{Assessment and Quantification of Effective Communication}

Many assessment methods and instruments were reviewed. While many instruments exist to assess effective communication skills, they appear to be created for a specific discipline or professional training course. For example Derkx, Rethans, Knottnerus, and Ram reported on an instrument they developed in which they established the validity and reliability that assesses essential communication skills among call handlers in out-of-hours centers. ${ }^{19}$ Among these individuals, it is vital to follow a particular course when handling medical inquiry calls. As a part of their accreditation, U.S. medical schools have developed instruments and methods to assess the communication skills of their students. Checklists (Likert type rating scales completed by faculty or other "raters"), surveys (completed by patients or receivers of communication), interviews, and essay exams are all commonly used methods in medical school curricula to assess communication. ${ }^{20}$ Various organizations and accreditation agencies of educational programs, colleges, and universities may also prescribe criteria that must be met regarding communication ability. Elements common to most of the instruments reviewed were the generic abilities of listening, speaking, and writing in the required language. 19,20 The use of "standardized patients" (actors presenting a scripted condition) have also become a preferred method of assessing communication skills in medical schools. ${ }^{21}$ 
There seems to be a wide variety of criteria and lack of consistency in disciplines outside of medical schools. Kimberlin examined U.S. pharmacy schools and found that communication skills in general were being taught and assessed but that the criteria lacked consistency.22 Other instruments assessing communication skills in undergraduate education were also reviewed, including the Communication Competence Self-Report (CCSR), Basic Course Communication Competency Measure, and the Communication Adaptability Scale (CAS). It was felt that these instruments focused primarily on core or entry level communication skills. While this is important to assess, the selective admissions process of many healthcare or human services programs suggests that a more specific set of communication skills be assessed. The necessary skill set should be somewhere between what is measured with the undergraduate instruments and the graduate instruments found in medical schools and other graduate programs. Additionally, the initial intent of this project was to develop an instrument to be used as an admission criterion for entrance into healthcare and human services programs, not "in program" progression of communication skills, although the results indicate that it has value for the latter.

\section{METHODS}

Approval was first obtained from Midwestern State University's IRB (approval number 10021603). A literature search was then conducted by a committee on three broad areas of communication: definition, essential elements, and methods of quantification. Relevant and representative articles were selected for each area to construct the literature review. Based on the findings of the literature review and the needs of the committee, a communication skills assessment instrument was created. To assess the desired elements of communication, an electronic format for the instrument was necessary. The format includes video scenarios and written scenarios, as well as self-report questions. The instrument was pilot tested and adjustments were then made to the scaling of the self-report section, to the scoring rubric to clarify intent, and to essential elements of the video and written segments. A case study design was applied with participants solicited from the College of Health Sciences and Human Services departments who also used a process to assess communication skills in some quantifiable way. The instrument was then administered to participants via Blackboard prior to beginning the class. Data were imported and analyzed using IBM SPSS Version 19.

\section{INSTRUMENT}

The instrument that was developed was based on the literature reviewed and consisted of three segments. The first segment consisted of two scenarios presented in video format to assess listening skills and recognition of verbal skills. To assess listening skills, the participant was asked to summarize the lay person's (actor in video) issue or problem that he or she was attempting to convey. To assess recognition of verbal skills, the participant was asked to answer two questions regarding what the professional (actor in video) did incorrectly or inappropriately. The four constructs assessed were: articulation, listening comprehension, conversation skills, and sensitivity-prejudice.

The second segment consisted of an 18-item self-report section assessing the participants' perceived verbal and nonverbal skills. The first nine items focused on verbal skills and the remaining nine on nonverbal skills. This segment asked the participant to indicate the degree to which a statement applied to him/her. A 4-point Likert scale was used as follows: 1=strongly describes me, 2=moderately describes me, $3=$ slightly describes me, 4=does not describe me at all. Eleven of these items were rescaled during analysis.

The third segment presented two scenarios in written form, each of which contained four nonverbal expressions, both appropriate and inappropriate, for the participant to identify. The first scenario addressed the constructs of: body movement/posture, voice, facial expression, and eye contact. The second scenario addressed the constructs of proxemics, eye contact, voice inflection, and patronizing touch. The participant's writing ability was also evaluated for content, clarity, organization, and grammar.

The maximum number of points possible was 96 . The most desirable answer on the self-report section was awarded 4 points, the second 3 points, and so on. Again, 11 items were reverse scaled to keep the point awards correct. The first and third segments were hand scored by the researcher using the established scoring rubric. All scores were then entered into IBM SPSS Version 19 for analysis.

\section{RESULTS}

Correlations were first conducted to determine if any correlations existed within the segments of the instrument. The video segments and written segments were significantly correlated $(r=.269$ and significant at the 0.01 level 2-tailed). No significant relationships were found between the self-report segment and the other two. Three departments initially began the study: radiologic technology, respiratory care, and nursing. These departments had in place an instrument to measure effective communication skills that could be converted and compared to our instrument. Because participation was voluntary, the only 
department with sufficient numbers to analyze was nursing $(n=89)$. This department used the Interpersonal Process Recall (IPR) instrument early in their education process as a pre- and post-test evaluation in a communication skills course. Our communication instrument was administered prior to beginning the course and results compared to the IPR pre-test scores. These participants were isolated and their instrument scores and data from their IPR instrument were compared.

Data were analyzed using IBM SPSS Version 19. Linear regression correlation tests were conducted. A communication instrument composite score was first calculated for each participant after the appropriate items were reverse-scaled. The pre-test scores were also obtained from the nursing department for each participant on the IPR instrument. Both sets of scores were then converted to $z$ scores so that the same metric could be compared. A bivariate linear regression analysis was conducted to evaluate whether our communication instrument predicted performance on the IPR. The scatterplot (figure 1) indicates that the two variables are linearly related in that as the communication instrument score increases so does the IPR score. The $95 \%$ confidence interval for the slope, 0.485 to 0.832 does not contain the value of zero, thus the communication instrument scores significantly correlate to IPR scores. The accuracy of the communication instrument in predicting IPR scores is high $(r=.62)$. Approximately $39 \%$ of the variance in IPR scores was accounted for by its linear relationship with the communication instrument.

Significant correlations were also found between our communication instrument and those who indicated English was their second language, and between the nursing IPR instrument and those who indicated English was their second language. That is, those who indicated English was their second language had lower scores on our instrument and the IPR.

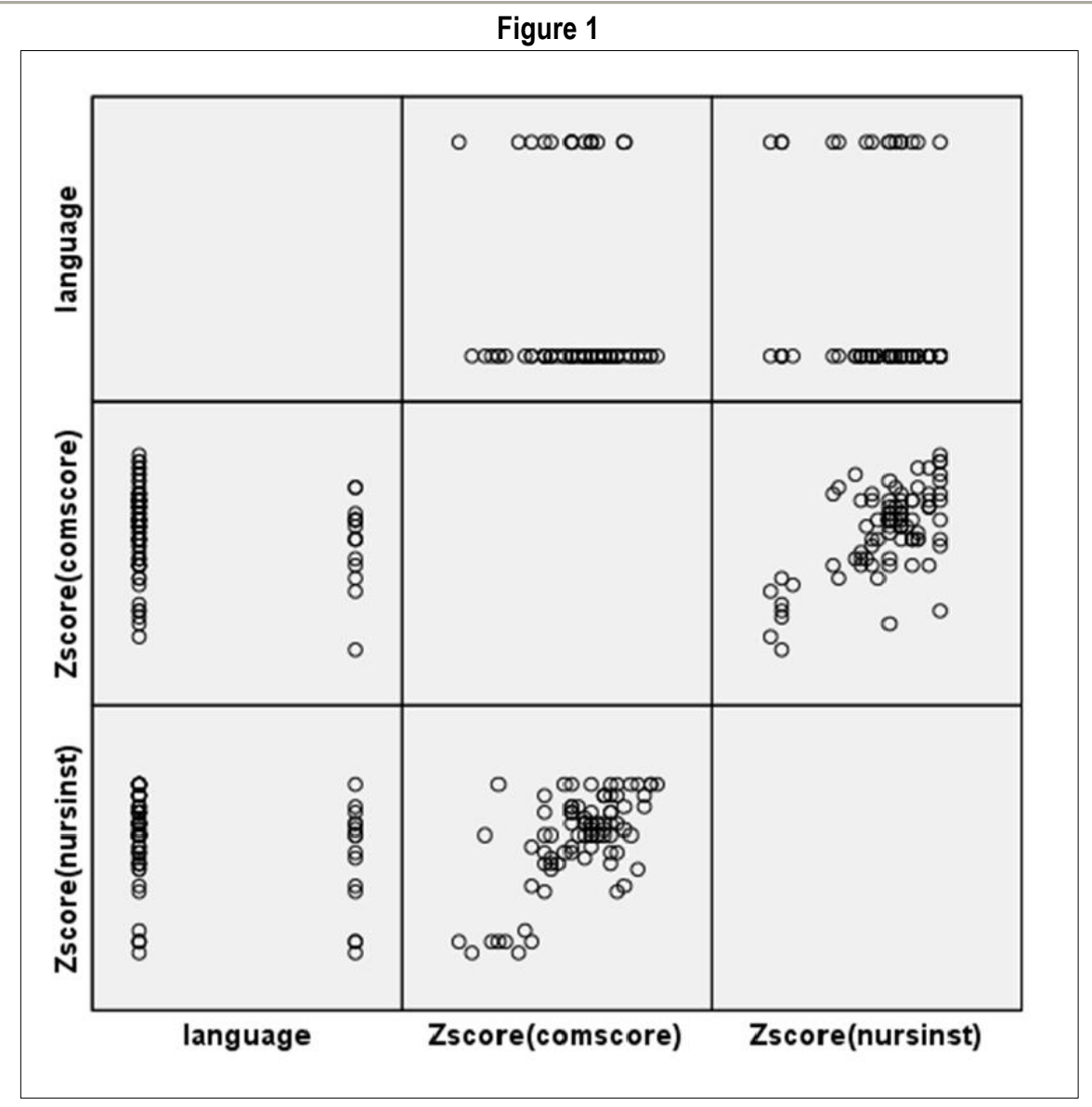

\section{DISCUSSION}

This project began as a result of the experiences by several departments in a College of Health Sciences and Human Services, where students had problems with communication skills after entering the clinical or field experience phase of training. These departments also reported moderate success in improving these skills at that point. As previously stated, research also indicates that communication skills do not necessarily improve with experience. ${ }^{7}$ Because of the competitive nature and selective admission process of many healthcare and human services programs, it is critical to select applicants with the greatest potential 
for success so as not to "waste" a position in that program's cohort. There are already a number of criteria in use for selecting applicants such as GPA, aptitude test scores, ACT and/or SAT scores, personal interview, etc., and yet in our experiences, this communication problem persists. The goal of this project was to develop an instrument that could identify a core set of communication skills that may be built upon once accepted into a healthcare or humans services program. This would directly address the issue of poor communication skills in the clinical phase of training mentioned above. Our instrument appears to have value in assessing such skills.

The instrument may be used as an additional tool for selection of healthcare professionals. Using the instrument as part of an admission process may help increase retention. The majority of healthcare and human service education programs experience high attrition rates. ${ }^{23}$ As previously mentioned, most commonly programs use GPA and standardized entrance exams for admission criteria, but there are no universally accepted admission requirements. ${ }^{23}$ Most healthcare and human service programs include didactic and clinical aspects. This provides a unique set of conditions compared to other traditional college programs when looking at the reliability of admission requirements. If communication skills are a part of the attrition problem and the instrument can predict performance in this area, then poor performers could be advised into other fields reducing attrition from this cause. This instrument may then help bridge the gap of attrition and retention for healthcare and human service education programs. Another potential use of this instrument is as a pre- and post-test assessment of courses or modules of instruction intended to improve intercommunication skills (although this was not originally anticipated). The instrument could first be used to help select those with the greatest potential for success then used as a pre and post- test outcomes measure for assessing improvement in communication skill within the professional program of study. Further research is needed to establish a minimum score on the instrument that indicates an acceptable communication skill level. Additionally, longitudinal studies are needed to compare predicted communication skill to actual clinical performance.

\section{REFERENCES}

1. David RA, Rhee M. The impact of language as a barrier to effective health care in an underserved urban Hispanic community. Mount Sinai Journal of Medicine. 1998;65(5-6):393-7. [PMID 9844369]

2. Leonard M, Graham S, Bonacum D. The human factor: The critical importance of effective teamwork and communication in providing safe care. Quality and Safety in Health Care. 2004;13(Suppl 1):i85-90. Available at: www.inti-qhc.bmijournals.com . Accessed September 11, 2006. [PMID 15465961]

3. Buller MK, Buller DB. Physicians' communication style and patient satisfaction. Journal of Health and Social Behavior. 1987;28(4):375-88. [PMID 3429807]

4. Mandl KD, Kohane IS, Brandt AM. Electronic patient-physician communication: Problems and promise. Ann Intern Med. 1998;129(6):495-500. [PMID 9735088]

5. Paling J. Strategies to help patients understand risks. Br Med J.2003;327(7417);745-8. [PMID 14512489]

6. Fallowfield LJ, Jenkins VA. Effective communication skills are the key to good cancer care. Eur J Cancer. 1999;35(1):15927. [PMID 10673967]

7. Moore PM, Wilkinson SM, Rivera Mercado S. Communication skills training for health care professionals working with cancer patients, their families and/or careers. Cochrane Database System Rev. 2004;(2),CD003751.

8. van der Laan J M. Plastic words: Words without meaning. Bulletin of Science, Technology \& Society. 2001;21(5):349.

9. Pei M. The Story of Language, Revised Ed. J.B. Lippincott Company, NY,1965.

10. Bezemer J, Kress $G$. Writing in multimodal texts: A social semiotic account of designs for learning. Written Communication. 2008;25(2):166-95. DOI:10.1177/0741088307313177.

11. Ogili E. Strategies for effective communication of educational instructions in Nigeria. Malaysian Online J of Instructional Technol (MOJIT). 2005;2(1):1-7.

12. Grover SM. Shaping effective communication skills and therapeutic relationships at work: The foundation of collaboration. AAOHN J. 2005;53(4):177-182.[15853294]

13. le Roux J. Effective educators are culturally competent communicators. Intercultural Ed. 2002;13(1):37-48. DOI:10.1080/14675980120112922.

14. Makoul G. Essential elements of communication in medical encounters: The Kalamazoo consensus statement. Acad Med. 2001;76(4):390-3. [PMID 11299158]

15. Sharpe DL, Anderson C, White A, Galvan S, Siesta M. Specific Elements of Communication that affect trust and commitment in the financial planning process. Financial Counseling and Planning. 2007;18(1):2-17.

16. De-hua W, Hui L. Nonverbal language in cross-cultural communication. US-China Foreign Language. 2007;S(10):66-70. Available at: http://www.cqvip.com/qk/88585x/2007010/25808062.html . Accessed April 17, 2009.

17. Dyche LJ. Interpersonal skill in medicine: The essential partner of verbal communication. Gen Intern Med. 2007;22(7):10359. [PMID 17437144] 
18. Griffith $\mathrm{CH}$, Wilson JF, Langer S, Haist SA. House staff nonverbal communication skills and standardized patient satisfaction. J Gen Int Med. 2003;18(3):170-4. DOI: 10.1046/j.1525-1497.2003.10506.x

19. Derkx HP, Rethans JJ, Knottnerus JA, Ram PM. Assessing communication skills of clinical call handlers working at an outof-hours centre: Development of the RICE rating scale. Br J Gen Pract. 2007;57(538):383-7.

20. Duffy FD, Gordon GH, Whelan G, Cole-Kelly K, Frankel R, Buffone N, et al. Assessing competence in communication and interpersonal skills: the Kalamazoo II report. Acad Med. 2004;79(6):495-507.

21. Fernandez A, Wang F, Braveman M, Finkas L, Hauer KE. Impact of student ethnicity and primary childhood language on communication skill assessment in a clinical performance examination. J Gen Int Med. 2007;22(8):1155-60. [PMID 17558538]

22. Kimberlin CL. Communicating with patients: Skills assessment in US colleges of pharmacy. Am J of Pharm Ed. 2006;70(3):67. [PMID 17136187]

23. Espen D, Wright D, Killion JB. Admission Requirements for Radiography Programs. Radiologic Technol. 2006;77:366-72.

\section{KEY TERMS}

Communication Skills, Effective Communication, Healthcare and Human Service Admission 